DOI: $10.15593 / 2224-9982 / 2020.63 .08$

УДК 681.5.015.44

\author{
А.А. Иноземцев ${ }^{1,2}$, Н.Г. Ламанова ${ }^{1}$, А.Н. Саженков ${ }^{2}$, \\ И.Н. Грибков ${ }^{2}$, А.С. Плешивых ${ }^{1,2}$ \\ ${ }^{1}$ Пермский национальный исследовательский политехнический университет, Пермь, Россия \\ ${ }^{2}$ ОДК-Авиадвигатель, Пермь, Россия

\section{СИНТЕЗ ОПТИМАЛЬНОГО НАБЛЮДАТЕЛЯ ПРИ ОТСУТСТВИИ АПРИОРНОЙ ИНФОРМАЦИИ О ХАРАКТЕРИСТИКАХ ШУМА ВОЗМУЩЕНИЯ СИСТЕМЫ АВТОМАТИЧЕСКОГО УПРАВЛЕНИЯ И КОНТРОЛЯ ТУРБОРЕАКТИВНОГО ДВУХКОНТУРНОГО ДВИГАТЕЛЯ}

\begin{abstract}
Возможность получения измерительной информации о параметрах системы автоматического управления и контроля турбореактивного двухконтурного двигателя при его функционировании в условиях полета и практически отсутствие ограничений по вычислительным затратам позволяют использовать алгоритмические методы повышения отказоустойчивости САУ и К двигателя.

В предшествующей авторской работе в качестве резервного измерителя использовался оптимальный наблюдатель - фильтр Калмана. Его функционирование существенно зависит от вероятностных характеристик системного шума, который известен приблизительно и изменяется сложным образом во времени. Неточность этих характеристик может привести к расходимости фильтра и потере его устойчивости.

Для решения этой проблемы предлагается использовать адаптивный фрильтр Язвинского, который позволяет вычислять ковариационную матрицу шума возмущения после поступления новых измерений по значениям обновляемого процесса фильтра Калмана. При этом вычислительные затраты увеличиваются незначительно.

Использование адаптивного фильтра Язвинского исключает необходимость предварительного корреляционного анализа системного шума и защищает фильтр Калмана от потери устойчивости.

Представлены результаты моделирования фильтра Язвинского, согласованного с математической моделью ТРДД, по данным летных испытаний двигателя типа ПС-90А в составе самолета Ту-214 как на стационарном, так и на переходном режиме работы двигателя. Параметры указаны в процентах от максимальных значений.

Проведен сравнительный анализ фрактических ошибок оценивания вектора выхода САУ и К ТРДД при работе фильтров Калмана и Язвинского.

Результаты сравнительного анализа представлены в виде таблиц и графиков.

Показано, что предложенный алгоритм обеспечивает выполнение требований по точности определения оценок вектора выхода системы автоматического управления и контроля газотурбинного двигателя и может быть рекомендован для использования в САУ и К ТРДД.

Ключевые слова: математическая модель, отказоустойчивость, оптимальные оценки, фильтр Калмана, фильтр Язвинского, шум возмущения.
\end{abstract}

\author{
A.A. Inozemtsev ${ }^{1,2}$, N.G. Lamanova ${ }^{1}$, A.N. Sazhenkov², \\ I.N. Gribkov ${ }^{2}$, A.S. Pleshivikh ${ }^{1,2}$ \\ ${ }^{1}$ Perm National Research Polytechnic University, Perm, Russian Federation \\ ${ }^{2}$ UEC-Aviadvigatel, Perm, Russian Federation
}

\title{
SYNTHESIS OF THE OPTIMAL OBSERVER IN THE ABSENCE OF APRIORI INFORMATION ABOUT THE NOISE CHARACTERISTICS OF THE AUTOMATIC CONTROL AND MONITORING SYSTEM OF A TURBOFAN ENGINES
}

The possibility of obtaining measuring in-formation about the parameters of the automatic control and monitoring system of a turbofan engine during its operation in flight conditions and almost no restrictions on computational costs enable using algorithmic methods to improve the engine fault tolerance.

In the previous article, the optimal observer, the Kalman filter, was used as a reserve meter. Its functioning essentially depends on the probabilistic characteristics of the system noise, which is known approximately and changes in a complex manner over time. Inaccuracies in these characteristics can lead to filter divergence and loss of its stability. 
To solve this problem, it is proposed to use the Yazvinsky adaptive filter, which allows calculating the covariance matrix of the disturbance noise after the arrival of new measurements from the values of the updated process of the Kaman filter. In this case computational costs increase insignificantly.

The use of the Yazvsky adaptive filter eliminates the need for preliminary correlation analysis of system noise and protects the Kalman filter from loss of stability.

The results of modeling the Yazvinsky filter, consistent with the mathematical model of the turbofan engine, based on the flight test data of the PS-90A type engine as part of the TU-214 aircraft, both in steady-state and transient modes of engine operation, are presented. The parameters are indicated as percentage of the maximum values.

A comparative analysis of the actual errors in the estimation of the output vector of the turbofan engine ACS when Kalman and Yazvinsky filters are operating is carried out.

The results of the comparative analysis are presented in the form of tables and graphs.

It is shown that the proposed algorithm ensures fulfillment of the requirements for the accuracy of estimating the output vector of the automatic control and monitoring system of a gas turbine engine and can be recommended for use in the ACS of the turbofan engine.

Keywords: mathematical model, failure tolerance, optimal estimates, Kalman filter, Yazvinsky filter, disturbance noise.

Для обеспечения отказоустойчивости системы автоматического управления и контроля (САУ и К) турбореактивного двухконтурного двигателя (ТРДД) необходима информационная избыточность, которая может быть реализована при помощи физического или аналитического резервирования. При аналитическом резервировании используются алгоритмические методы. Так, в предшествующей авторской работе [1] рассматривался метод, в котором в качестве резервного измерителя применялся оптимальный фильтр Калмана (ОФК). Для его функционирования, кроме адекватной математической модели объекта управления, необходимо знать вероятностные характеристики случайных возмущений, действующих на систему управления, и случайных ошибок измерения сигналов датчика $[2,3]$.

К внешним воздействиям можно отнести всевозможные случайные помехи, характеристики каналов распространения сигналов, неустранимые шумы квантования и др. В реальных условиях входные воздействия известны приблизительно и изменяются во времени сложным образом [4]. Поскольку спектр входных воздействий полностью не определен, не представляется возможным получить статистически достоверные характеристики системных шумов. При этом известно [5], что они оказывают существенное влияние на процесс фильтрации. Неточность характеристик системных шумов может привести к расходимости фильтра, которая выражается в том, что фактические ошибки оценивания вектора состояния системы управления значительно превышают ошибки, прогнозируемые фильтром. В работе [1] при анализе системных шумов учитывались только возмущения, вызы- ваемые флуктуацией дозирующей иглы при подаче топлива в камеру сгорания ТРДД.

Измерительные шумы, вызываемые ошибками измерения сигналов, влияют на процесс оценивания вектора выхода САУ и К ТРДД в меньшей степени, а результаты их анализа более статистически достоверны, поскольку точностные характеристики измерителей, как правило, известны и изменяются во времени незначительно.

Эти обстоятельства приводят к необходимости использования адаптивных фильтров. Преимущество адаптивного оценивания заключается во введении в алгоритм механизма регулярного поиска оптимального значения оцениваемого параметра в пределах возможного класса решений $[6,7]$. Фильтр Калмана (ФК) не является в полной мере адаптивным, так как он формирует оптимальную оценку вектора состояния при поступлении новых измерений только при условии, что известны характеристики шумов [8]. Адаптивным является фильтр Язвинского (ФЯ), который генерирует оптимальную оценку при отсутствии информации о вероятностных характеристиках системных шумов.

А.Г. Язвинский (А.Н. Jazwinski) предложил подход к разработке адаптивных фильтров, позволяющий оценить неизвестную ковариационную матрицу шума возмущения после получения значений обновляемых процессов ФК [9]. Алгоритм ФЯ представляет собой алгоритм ОФК, в который встроена процедура вычисления матрицы $Q(k)$ по мере поступления новых измерений. Возможность оценивания характеристик системных шумов в процессе фильтрации повышает степень адаптивности ОФК. 
Математическая модель ТРДД может быть представлена в виде $[1,10,11]$

$\left\{\begin{array}{c}x(k+1)=\Phi x(k)+\Psi u(k)+\Phi_{0}+w(k) \\ z(k+1)=H x(k+1)+D u(k+1)+H_{0}+v(k+1)\end{array}\right.$

где $x(k)-$ вектор состояния, $\quad x(k)=$ $=\left[n_{\mathrm{в}}(k) \quad n_{\mathrm{\kappa}}(k)\right]^{T} ; u(k)-$ вектор управляющего воздействия, $u(k)=\left[\begin{array}{ll}G_{\mathrm{T}}(k) & \varphi_{\text {вна }}(k)\end{array}\right]^{T} ; z(k)-$ вектор измерений (выхода), $z(k)=$ $=\left[\begin{array}{ll}z_{1}(k) & z_{2}(k)\end{array}\right]^{T} ; z_{1}(k)$ - вектор прямых измерений вектора состояния, $z_{1}(k)=$ $=\left[n_{\mathrm{B}}{ }^{u}(k) \quad n_{\mathrm{K}}{ }^{u}(k)\right]^{T} ; \quad z_{2}(k)-$ вектор косвенных измерений, $\quad z_{2}(k)=\left[P_{\mathrm{K}}(k) T_{\mathrm{T}}(k)\right]^{T}$; $w(k)$ - вектор шума возмущения, $w(k)=\left[\begin{array}{ll}w_{1}(k) & w_{2}(k)\end{array}\right]^{T} ; v(k)$ - вектор шума измерений, $\quad v(k)=\left[\begin{array}{llll}v_{1}(k) & v_{2}(k) & v_{3}(k) & v_{4}(k)\end{array}\right]^{T}$; $n_{\mathrm{B}}(k), n_{\mathrm{K}}(k)$ - частота вращения вала ротора низкого и высокого давления; $G_{\mathrm{T}}(k)$ - расход топлива; $\varphi_{\text {вна }}(k)$ - угол входных направляющих аппаратов; $P_{\kappa}(k)$ - давление газа за компрессором; $T_{\mathrm{T}}(k)$ - температура газа за турбинами; $\Phi, \Psi, \Phi_{0}, H, D, H_{0}$ - матрицы коэффициентов соответствующих размерностей.

Полагается, что $x(k), z(k), u(k)$ - гауссовские процессы, начальное состояние $x(0)$; $M\{x(0)\}=\bar{x} ; \quad M\left\{x(0) x^{T}(0)\right\}=P(0 / 0) ;$ $w(k), v(k)$ - центрированные гауссовские процессы; $M\{w(k)\}=M\{v(k)\}=0$; ковариационные матрицы системных и измерительных шумов; $\operatorname{cov}\{w(k)\}=M\left\{w(k) w^{T}(k)\right\}=Q(k)$, $\operatorname{cov}\{v(k)\}=M\left\{v(k) v^{T}(k)\right\}=R(k) ;$ дисперсионные матрицы: $\operatorname{diag} R=\left[\begin{array}{lll}\sigma_{v 1}{ }^{2} & \sigma_{v m}{ }^{2}\end{array}\right] ; \operatorname{diag} Q=$ $=\left[\begin{array}{lll}\sigma_{w 1} & \sigma_{w p}{ }^{2}\end{array}\right]$.

Из этих предположений следуют свойства $[5,12]$ :

$$
\begin{aligned}
& M\left\{x(i) w^{T}(j)\right\}=0 \text { для } j \geq i, i=0,1, \ldots ; \\
& M\left\{z(i) w^{T}(j)\right\}=0 \text { для } j \geq i, i=1,2, \ldots ; \\
& M\left\{x(i) v^{T}(j)\right\}=0 \text { для любых } j \text { и } i ; \\
& M\left\{z(i) v^{T}(j)\right\}=0 \text { для любых } j>i .
\end{aligned}
$$

В классическом фильтре модель измерений имеет вид

$$
z(k+1)=H x(k+1)+v(k+1) .
$$

Обновляемый процесс ФК $\tilde{z}(k+1 / k)-$ ошибка прогнозной оценки вектора измерений $z(k+1)$ :

$$
\tilde{z}(k+1 / k)=z(k+1)-H \hat{x}(k+1 / k),
$$

где $\hat{x}(k+1 / k)$ - прогнозная оценка вектора состояния.

Обновляемый процесс дает информацию как о текущих, так и о предшествующих измерениях, поступающих в фильтр.

Введем обозначение $\tilde{z}(k+1 / k)=v(k+1)$.

Поскольку ковариационная матрица шума системы $Q(k)$ зависит от значений обновляемого процесса ФК, изменяется последовательность обработки измерений, поступающих в фильтр [1], и алгоритм фильтрации принимает вид

$$
\begin{aligned}
& \int \hat{x}(k+1 / k)=\Phi \hat{x}(k / k)+\Psi u(k)+\Phi_{0} ; \\
& \mathrm{v}(k+1)=z(k+1)-H \hat{x}(k+1 / k) \text {; }
\end{aligned}
$$

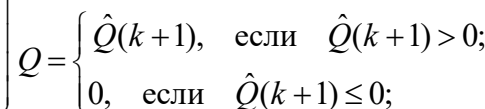

$$
\begin{aligned}
& P(k+1 / k)=\Phi P(k / k) \Phi^{T}+\Gamma Q \Gamma^{T} \\
& K(k+1)=P(k+1 / k) H^{T}\left[H P(k+1 / k) H^{T}+R(k+1)\right]^{-1} ; \\
& P(k+1 / k+1)=[E-K(k+1) H] P(k+1 / k) \text {; } \\
& \hat{x}(k+1 / k+1)=\hat{x}(k+1 / k)+K(k+1) \cup(k+1),
\end{aligned}
$$

где $z(k+1)-$ новое измерение; $\hat{x}(k+1 / k+1)-$ оптимальная оценка вектора состояния; $P(k+1 / k), P(k+1 / k+1)$ - ковариационные матрицы ошибок прогноза и фильтрации соответственно; $K(k+1)$ - матричный коэффициент усиления фильтра.

Оценка фильтрации удовлетворяет следующим свойствам [5]:

$$
\begin{aligned}
& \qquad M\left\{\tilde{x}(k / k) z^{T}(l)\right\}=0 \text { для } l \leq k ; \\
& M\{\hat{x}(k / k)\}=M\{\hat{x}(k+1 / k)\}=M\{x(k)\},(6) \\
& \text { где } \tilde{x}(k / k)-\text { ошибка фильтрации, } \tilde{x}(k / k)= \\
& =x(k)-\hat{x}(k / k) .
\end{aligned}
$$



мулы (3)

Обновляемый процесс с учетом фор-

$$
\begin{gathered}
v(k+1)=H x(k+1)+v(k+1)-H \hat{x}(k+1 / k)= \\
=H[x(k+1)-\hat{x}(k+1 / k)]+v(k+1) ; \\
v(k+1)=H \widetilde{x}(k+1 / k)+v(k+1),
\end{gathered}
$$

где $\widetilde{x}(k+1 / k)-$ ошибка прогнозной оценки вектора состояния.

При определении ковариационной матрицы шума системы решающее значение имеет ковариационная матрица обновляемого процесса

$$
\operatorname{cov}\{v(k+1)\}=M\left\{v(k+1) v^{T}(k+1)\right\},
$$

которая в соответствии с методом моментов приравнивается к выборочной ковариационной матрице

$$
\begin{aligned}
& M\left\{v(k+1) v^{T}(k+1)\right\}=\hat{M}\left\{v(k+1) v^{T}(k+1)\right\} ; \\
& \hat{M}\left\{v(k+1) v^{T}(k+1)\right\}=v^{*}(k+1) v^{* T}(k+1),
\end{aligned}
$$

где $\mathrm{U}^{*}(k+1)$ - реализация обновляемого процесса.

Тогда

$$
\begin{aligned}
& M\left\{\cup(k+1) \cup^{T}(k+1)\right\}= \\
& =v^{*}(k+1) v^{* T}(k+1) .
\end{aligned}
$$

Из формул (2) и (6) вытекают свойства обновляемого процесса, которые учитываются при определении $\operatorname{cov}\{v(k+1)\}$ :

$$
\begin{gathered}
M\{\cup(k)\}=0 ; \\
M\{\tilde{x}(k / k) \cup(l)\}=0 \quad \text { при } l<k ; \\
M\left\{\cup(k) \cup^{T}(l)\right\}=0 \quad \text { при } k \neq l .
\end{gathered}
$$

Обновляемый процесс (7) является взвешенным средним двух статистически независимых векторных оценок, одна из которых $\widetilde{x}(k+1 / k)$, а вторая $-v(k+1)$ [13]. Ковариационные матрицы этих оценок соответственно

$$
\begin{aligned}
& \operatorname{cov}\{\tilde{x}(k+1 / k)\}=P(k+1 / k) ; \\
& \operatorname{cov}\{v(k+1)\}=R(k+1) .
\end{aligned}
$$

Тогда с учетом формул (10) и (11) теоретическая ковариационная матрица обновляемого процесса

$$
\begin{gathered}
\operatorname{cov}\{u(k+1)\}=M\left\{u(k+1) u^{T}(k+1)\right\}= \\
=H P(k+1 / k) H^{T}+R(k+1),
\end{gathered}
$$

где

$$
P(k+1 / k)=\Phi P(k / k) \Phi^{T}+\Gamma Q \Gamma^{T} .
$$

Таким образом, из формул (10) и (12) следует, что обновляемый процесс - это центрированный случайный процесс типа белого шума с нулевым математическим ожиданием и ковариационной матрицей (12).

После подстановки формул (12) и (13) в формулу (9) получим матричную систему уравнений

$$
\begin{gathered}
H \Phi P(k / k) \Phi^{T} H^{T}+H \Gamma Q \Gamma^{T} H^{T}+R(k+1)= \\
=v^{*}(k+1) v^{* T}(k+1),
\end{gathered}
$$

решением которой является выражение для вычисления искомой матрицы шума возмущения (системы) [14]:

$$
\begin{gathered}
\hat{Q}(k+1)= \\
=\frac{(H \Gamma)^{T}\left[\mathrm{v}^{*}(k+1) \mathrm{v}^{* T}(k+1)-H \Phi P(k / k) \Phi^{T} H^{T}-R(k+1)\right] H \Gamma}{\left[(H \Gamma)^{T} H \Gamma\right]^{2}} .
\end{gathered}
$$

Уравнения (5) и выражение (14) составляют алгоритм адаптивного ФЯ. Матрица $Q(k+1)$ неотрицательная, так как это дисперсионная матрица.

Для определения ковариационной матрицы шума измерения $R(k+1)$ достаточно предварительно провести корреляционный анализ сигналов датчиков САУ и К ТРДД для типичных режимов работы двигателя.

Вычислительные затраты для определения матрицы $\hat{Q}$ незначительны, так как коэффициенты математической модели (1) и значения обновляемого процесса (4), от которых она зависит, уже определены в процессе идентификации [15] и калмановской фильтрации [1].

Возможность определения вероятностных характеристик системного шума в процессе функционирования ТРДД защищает фильтр Калмана от потери устойчивости и, следовательно, обеспечивает стабильность оценок фильтрации.

Оптимальное оценивание вектора выхода САУ и К ТРДД в реальном масштабе времени предполагает решение следующих задач: 
1) идентификацию математической модели ТРДД с обеспечением требуемой точности и времени запаздывания;

2) оценивание вероятностных характеристик шума возмущения (системы);

3) оценивание вектора выхода САУ и К ТРДД на основе калмановской фильтрации.

В более ранних работах решение этих задач было разнесено по времени $[14,16]$. Использовались пакетные методы оценивания, так как существовали ограничения по вычислительным затратам, а также отсутствовали достаточные массивы измерительной информации, необходимые для моделирования алгоритмов оценивания. Ввиду этого полученные результаты были недостаточно информативны и обоснованы.

В настоящее время эти ограничения устранены. Появилась возможность использования рекуррентных методов оценивания математической модели и вектора выхода САУ и К ТРДД. Предлагаемая работа совместно с предшествующими работами $[1,15]$ позволяет совместить выполнение всех этапов оценивания в реальном масштабе времени при функционировании САУ и К ТРДД в реальных условиях эксплуатации и, следовательно, получить более статистически обоснованные оценки вектора выхода САУ и К ТРДД.

Реализация предложенного алгоритма проводилась в среде MATLAB с использованием аппаратной платформы со следующими параметрами: процессор Intel Core i7 2,67 ГГц, О3У 12 Гб.

Для расчета ФЯ потребовалось 0,695 с, ФК - 0,601 с, объем рабочего кода составил 3,6 и 3,52 Мб соответственно.
Моделирование ОФК и ФЯ, согласованных с математической моделью ТРДД, проводилось на основе информации датчиков САУ и К, полученной в результате летных испытаний двигателя типа ПС-90А в составе самолета Ту-214 в декабре 2018 г. на стационарных и переходных режимах работы двигателя, подробно изложенных в работе [1].

Рассматривались ФК и ФЯ, работающие на прямых измерениях вектора состояния. Результаты представлены в виде таблиц и графиков. Параметры ТРДД указаны в процентах от максимальных значений.

В табл. 1 приведены результаты анализа фактических ошибок оценивания вектора выхода САУ и К ТРДД на основе ФК и ФЯ на установившемся режиме [17].

На рис. 1 показаны процессы изменения сигналов выхода САУ и К ТРДД и соответствующие оптимальные оценки при работе ФК и ФЯ на установившемся режиме.

На рис. 2 показан процесс изменения матрицы $\hat{Q}$ на установившемся режиме.

Для оценивания качества оценок вектора выхода САУ и К ТРДД определены их фактическое смещение и стандартные ошибки оценивания: абсолютное фактическое смещение оценок фильтрации ФЯ на стационарном режиме составляет $(-0,1614-0,7650) \%$, ФК $(-0,4476-0,0021) \%$, среднеквадратическое отклонение вектора выхода САУ и К ТРДД ФЯ на стационарном режиме $(-0,1026-0,0293) \%$, ФК - $(0,0010-0,0110) \%$. Из анализа результатов оценивания качества следует, что ошибки оценивания вектора выхода САУ и К ТРДД ФК и ФЯ отличаются незначительно.

Таким образом, результаты анализа качества полученных оценок вектора выхода

Таблица 1

Результаты анализа фактических ошибок оценивания вектора выхода САУ и К ТРДД на основе ФК и ФЯ на установившемся режиме

\begin{tabular}{|c|c|c|c|c|}
\hline \multirow{2}{*}{$\begin{array}{c}\text { Оценка вектора } \\
\text { выхода } \\
\text { САУ и К ТРДД }\end{array}$} & $\begin{array}{c}\text { Смещение оценок } \\
\text { фильтрации, \% }\end{array}$ & $\begin{array}{c}\text { Среднеквадратическое } \\
\text { отклонение, \% }\end{array}$ & $\begin{array}{c}\text { Смещение оценок } \\
\text { фильтрации, \% }\end{array}$ & $\begin{array}{c}\text { Среднеквадратическое } \\
\text { отклонение, \% }\end{array}$ \\
\hline$\hat{n}_{\text {в }}$ & $-0,4252$ & 0,0010 & 0,7650 & 0,0293 \\
\hline$\hat{n}_{\text {к }}$ & $-0,4476$ & 0,0110 & $-0,1614$ & 0,0123 \\
\hline$\hat{P}_{\text {К }}$ & $-0,0996$ & 0,0026 & 0,3681 & 0,0086 \\
\hline$\hat{T}_{\mathrm{T}}$ & 0,0021 & 0,0032 & $-0,0025$ & $-0,1026$ \\
\hline
\end{tabular}



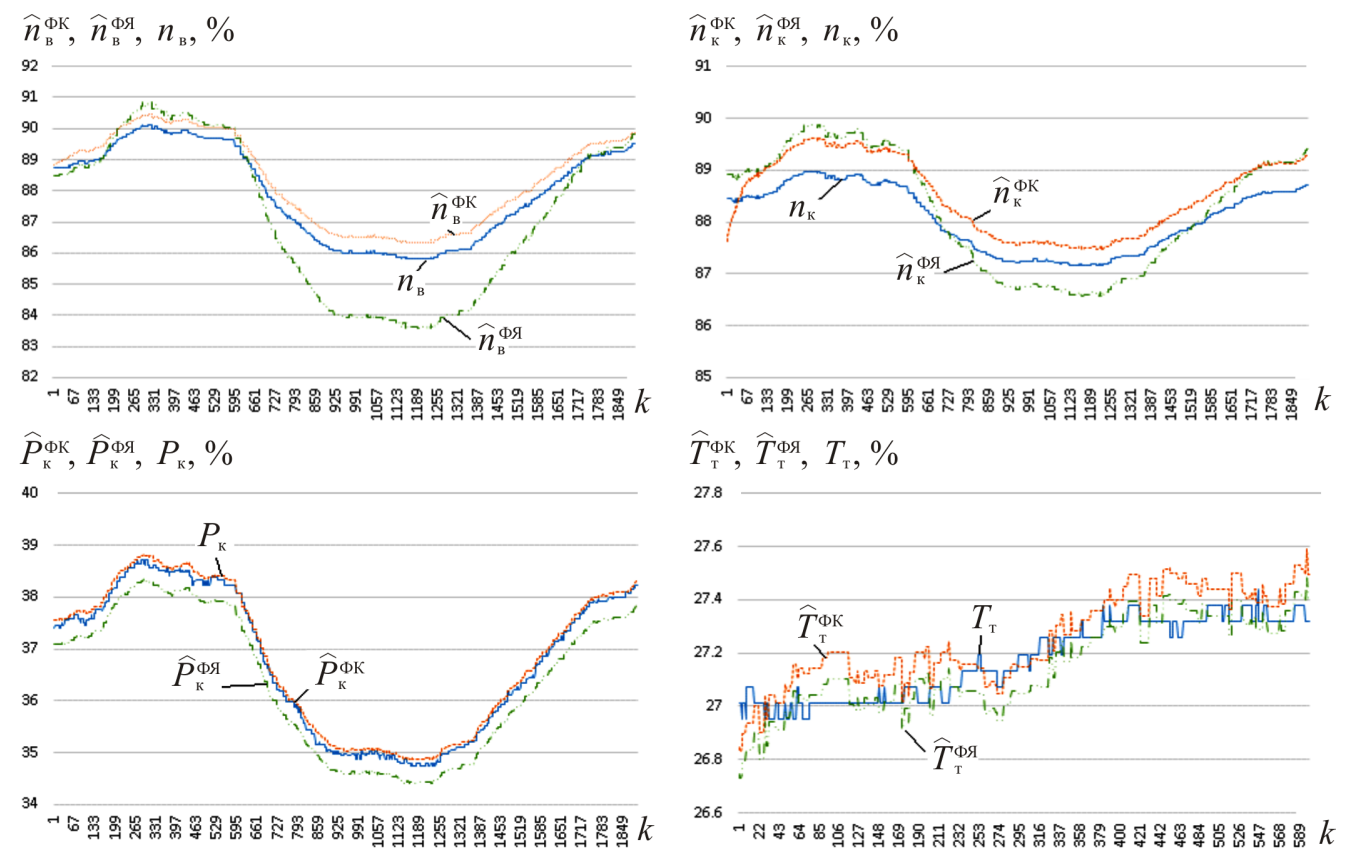

Рис. 1. Изменение сигналов выхода САУ и К ТРДД и соответствующие оптимальные оценки при работе ФК и ФЯ на установившемся режиме

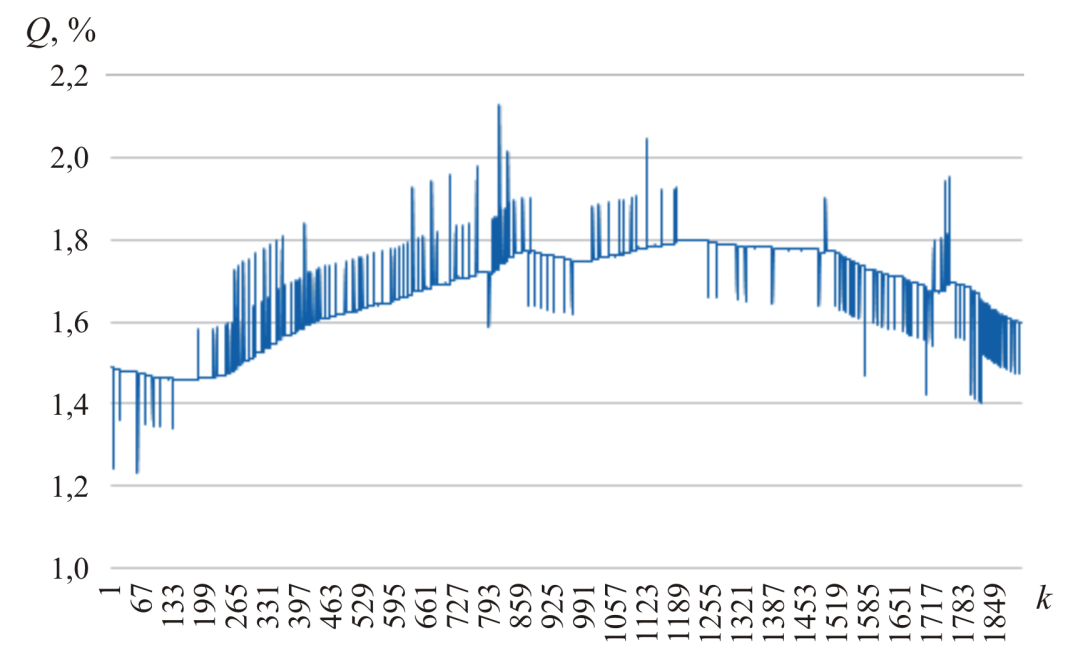

Рис. 2. Изменение матрицы $\hat{Q}$ на установившемся режиме

САУ и К ТРДД подтверждают работоспособность предложенного алгоритма адаптивной фильтрации фильтра Язвинского на стационарном режиме.

В табл. 2 приведены результаты анализа фактических ошибок оценивания вектора выхода САУ и К ТРДД на основе ФК и ФЯ на переходном режиме.

На рис. 3 показаны процессы изменения сигналов выхода САУ и К ТРДД и соответствующие оптимальные оценки при работе ФК и ФЯ на переходном режиме.

На рис. 4 показан процесс изменения матрицы $\hat{Q}$ на переходном режиме.
Для оценивания качества оценок вектора выхода САУ и К ТРДД определены их фактическое смещение и стандартные ошибки оценивания: абсолютное фактическое смещение оценок фильтрации ФЯ на переходном режиме составляет $(-3,1320-0,1431) \%$, ФК $(-13,1285-6,5209) \%, \quad$ среднеквадратическое отклонение вектора выхода САУ и К ТРДД ФЯ на переходном режиме $(0,0145-0,1169) \%$, ФК - $(0,0141-0,4341) \%$. Из анализа результатов оценивания качества следует, что стандартные ошибки отличаются незначительно, а смещение оценки ФЯ существенно меньше смещения оценки ФК. 
Таблица 2

Результаты анализа фактических ошибок оценивания вектора выхода САУ и К ТРДД на основе ФК и ФЯ на переходном режиме

\begin{tabular}{|c|c|c|c|c|}
\hline \multirow{2}{*}{$\begin{array}{c}\text { Оценка вектора } \\
\text { выхода }\end{array}$} & \multicolumn{2}{|c|}{ Фильтр Калмана } & \multicolumn{2}{|c|}{ Фильтр Язвинского } \\
\cline { 2 - 5 } САУ и К ТРДД & $\begin{array}{c}\text { Смещение оценок } \\
\text { фильтрации, \% }\end{array}$ & $\begin{array}{c}\text { Среднеквадратическое } \\
\text { отклонение, \% }\end{array}$ & $\begin{array}{c}\text { Смещение оценок } \\
\text { фильтрации, \% }\end{array}$ & $\begin{array}{c}\text { Среднеквадратическое } \\
\text { отклонение, \% }\end{array}$ \\
\hline$\hat{n}_{\text {в }}$ & $-13,1285$ & 0,4341 & $-3,1320$ & 0,1169 \\
\hline$\hat{n}_{\text {к }}$ & 6,5209 & 0,1790 & 0,1754 & 0,0613 \\
\hline$\hat{P}_{\text {К }}$ & $-0,0639$ & 0,1010 & 0,3210 & 0,1010 \\
\hline$\hat{T}_{\mathrm{T}}$ & 0,0430 & 0,0141 & 0,1431 & 0,0145 \\
\hline
\end{tabular}
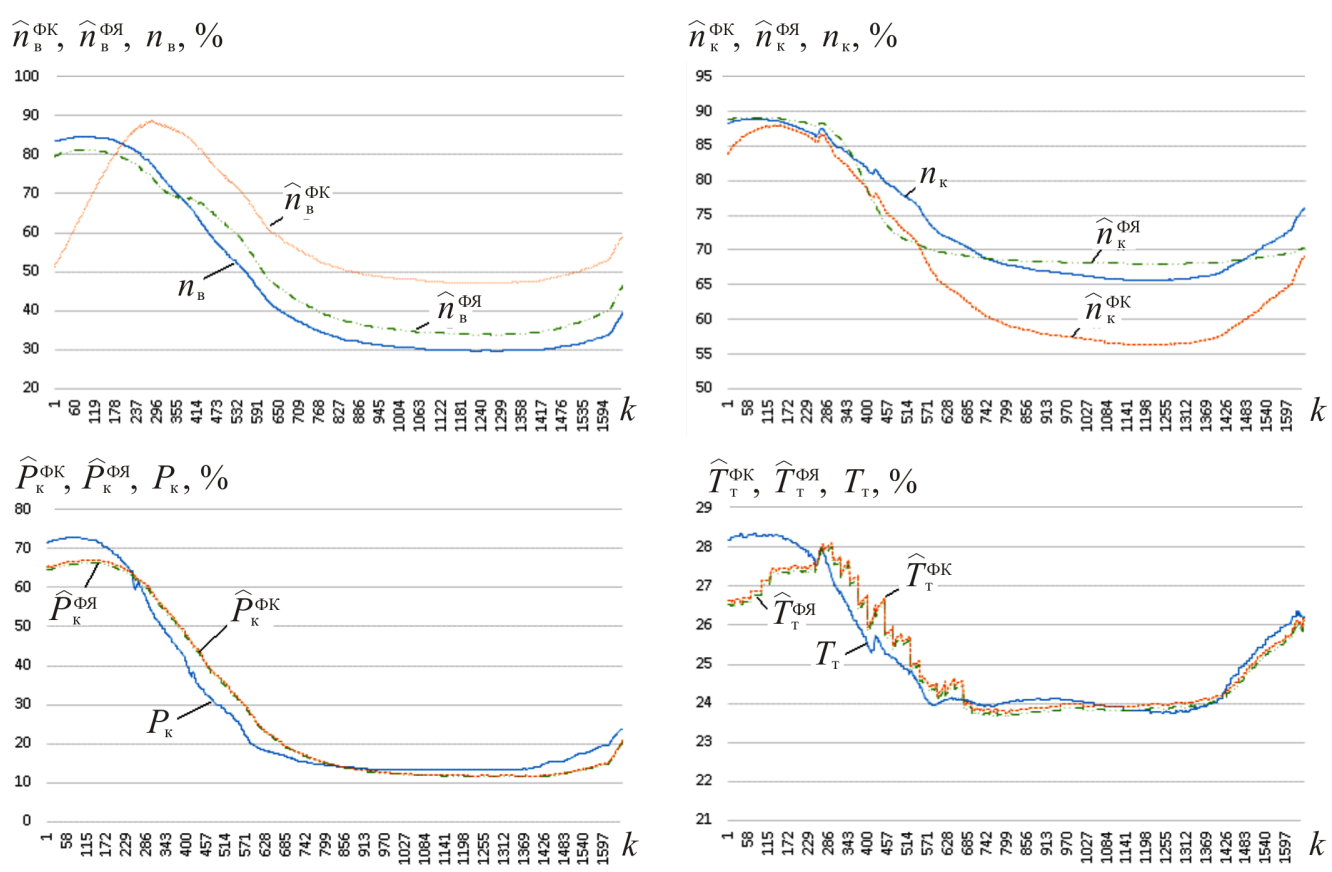

Рис. 3. Изменение сигналов выхода САУ и К ТРДД и соответствующие оптимальные оценки при работе ФК и ФЯ на переходном режиме

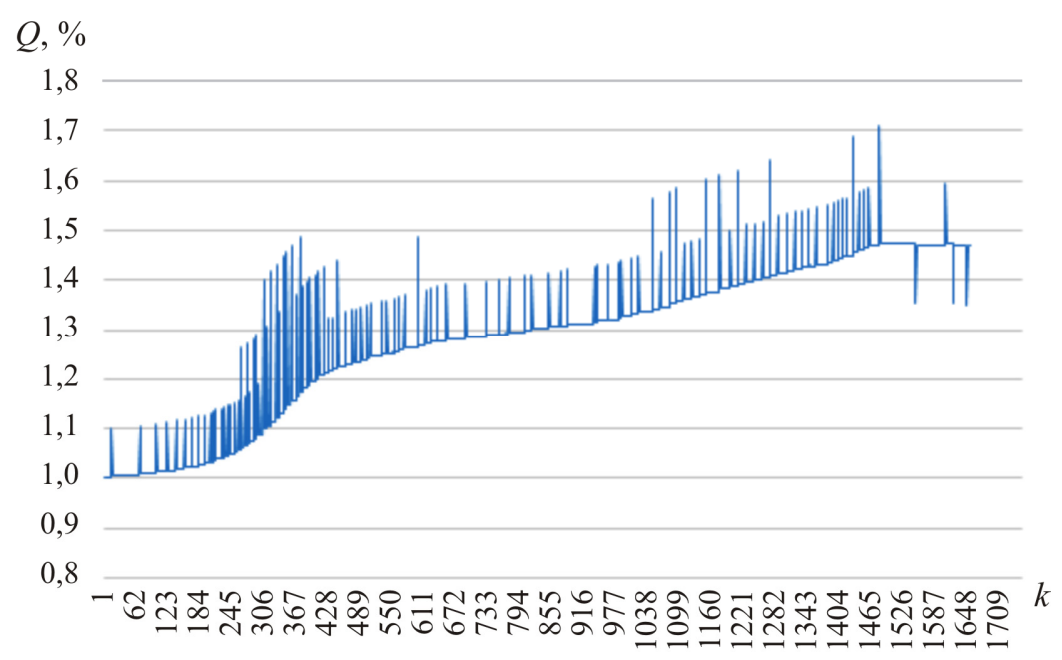

Рис. 4. Изменение матрицы $\hat{Q}$ на переходном режиме 
Таким образом, результаты анализа качества полученных оценок вектора выхода САУ и К ТРДД подтверждают работоспособность предложенного алгоритма адаптивной фильтрации фильтра Язвинского на переходном режиме.

Направление дальнейших исследований предусматривает математическое моделирование и сравнительный анализ ОФК и адаптивного ФЯ на динамических (экстремальных) режимах работы ТРДД и апробацию работы адаптивного ФЯ на стенде полунатурного моделирования с использованием технологий модельно-ориентированного проектирования [18, 19]. В качестве исследования планируется использовать авиационный двигатель пятого поколения ПД-14, применяемый для семейства российских самолетов типа МC-21.

\section{Заключение}

Моделирование оптимального фильтра Калмана и фильтра Язвинского, согласованных с математической моделью ТРДД, прово- дилось на основе информации датчиков САУ и К, полученной в результате летных испытаний двигателя типа ПС-90А в составе самолета Ту-214 на стационарных и переходных режимах работы двигателя.

Сравнительный анализ работы фильтра Калмана и фильтра Язвинского, согласованных с математической моделью ТРДД, показал, что ошибки оценивания вектора выхода САУ и К ТРДД отличаются незначительно как на стационарных, так и на переходных режимах. Однако при использовании адаптивного ФЯ возрастает стабильность оценок фильтрации, так как характеристики системного шума определяются в режиме реального времени, что снижает риски, связанные с возможной расходимостью ФК. Это преимущество позволяет рекомендовать адаптивный ФЯ для оценивания вектора выхода САУ и К ТРДД и использования его в современных САУ и К ТРДД.

\section{Библиографический список}

1. Синтез оптимального наблюдателя, согласованного с математической моделью САУ ТРДД / А.А. Иноземцев, Н.Г. Ламанова, А.Н. Саженков, И.Г. Лисовин, И.Н. Грибков, А.С. Плешивых // Вестник Пермского национального исследовательского политехнического университета. Аэрокосмическая техника. - 2019. - № 57. - С. 162-171.

2. Крамер Г. Математические методы статистики: пер. с англ. / под ред. А.Н. Колмогорова. - М.: Мир, 1975. - 648 с.

3. Развитие авиационных ГТД и создание уникальных технологий / В.И. Бабкин, М.М. Цховребов, В.И. Солонин, А.И. Ланшин // Двигатель. - 2013. - № 2 (86). - С. 6.

4. Бабкин В.И., Солонин В.И. Современная методология создания конкурентоспособных авиационных двигателей и место науки в этом процессе // Двигатель. - 2017. - № 1. - С. 10-13.

5. Медич Д. Статистические оптимальные линейные оценки и управление / под ред. А.С. Шаталова. М.: Энергия, 1973. - 440 с.

6. Адаптивные фильтры / под ред. К.Ф.Н. Коуэна и П.М. Гранта. - М.: Мир, 1988. - 440 с.

7. Сейдж Э.П., Уайт Ч.С. Оптимальное управление системами: пер. с англ. / под ред. Б.Р. Левина. М.: Мир, 1990. - 584 с.

8. Браммер К., Зиффлинг Г. Фильтр Калмана - Бьюси. Детерминированное наблюдение и стохастическая фильтрация. - М.: Наука, 1982. - 199 с.

9. Jazwinski A.H. Stochastic processes and filtering theory. - New York: Academic Press, 1970. $-376 \mathrm{p}$.

10. Марпл-мл. С.Л. Цифровой спектральный анализ и его приложения: пер. с англ. - М.: Мир, 1990. -584 c.

11. Интегральные системы автоматического управления силовыми установками самолетов / под ред. А.А. Шевякова. - М.: Машиностроение, 1983. - 283 с.

12. Кузовков Н.Т., Салычев О.С. Инерциальная навигация и оптимальная фильтрация. - М.: Машиностроение, 1982. - 216 с.

13. Изерман Р. Цифровые системы управления. - М.: Мир, 1984. - 541 с.

14. Ламанова Н.Г. Адаптивное оценивание вектора выхода САУ ГТД // Вестник Пермского государственного технического университета. Аэрокосмическая техника. - 2000. - № 4. - С. $37-42$.

15. Идентификация математической модели системы автоматического управления газотурбинного двигателя / Н.Г. Ламанова, А.С. Плешивых, И.Н. Грибков, А.И. Фатыков // Вестник Пермского нацио- 
нального исследовательского политехнического университета. Электротехника, информационные технологии, системы управления. - 2019. - № 31. - С. 121-135.

16. Ламанова Н.Г. Анализ математической модели САУ ГТД // Вестник Пермского государственного технического университета. Аэрокосмическая техника. - 1999. - № 2. - С. 76-81.

17. Теория автоматического управления силовыми установками летательных аппаратов / под ред. А.А. Шевякова. - М.: Машиностроение, 1976. - 344 с.

18. Скибин В.А., Солонин В.И., Палкин В.А. Работы ведущих авиадвигателестроительных компаний в обеспечение создания перспективных авиационных двигателей (аналитический обзор) / ЦИАМ. M., 2010. -673 c.

19. Плешивых А.С., Заборских А.А., Фатыков А.И. Стенд для испытаний электронной части систем автоматического управления газотурбинного двигателя // Вестник Пермского национального исследовательского политехнического университета. Электротехника, информационные технологии, системы управления. - 2017. - № 22. - С. 90-102.

\section{References}

1. Inozemcev A.A., Lamanova N.G., Sazhenkov A.N., Lisovin I.G., Gribkov I.N., Pleshivyh A.S. Sintez optimalnogo nablyudatelya, soglasovannogo s matematicheskoj modelyu SAU TRDD [Synthesis of the optimal observer, consistent with the mathematical model of the automatic control system of the turbojet engine]. PNRPU Aerospace Engineering Bulletin, 2019, no. 57, pp. 162-171.

2. Kramer G. Matematicheskie metody statistiki: Per. s angl [Mathematical methods of statistics]. Moscow: Mir, $1975,648 \mathrm{p}$.

3. Babkin V.I., Ckhovrebov M.M., Solonin V.I., Lanshin A.I. Razvitie aviacionnyh GTD i sozdanie unikalnyh tekhnologiy [Development of aviation gas turbine engines and creation of unique technologies]. Dvigatel, 2013, no. 2 (86), 6 p.

4. Babkin V.I., Solonin V.I. Sovremennaya metodologiya sozdaniya konkuren-tosposobnyh aviacionnyh dvigatelej i mesto nauki v etom processe. Dvigatel', 2017, № 1, pp. 10-13.

5. Medich D. Statisticheskie optimalnye linejnye ocenki i upravlenie [Statistical Optimal Linear Estimates and Control]. Moscow: Energiya, 1973, 440 p.

6. Kouen K.F.N., Grant P.M. Adaptivnye filtry [Adaptive filters]. Moscow: Mir, 1988, 440 p.

7. Sejdzh E.P., Uajt CH.S. Optimalnoe upravlenie sistemami: per. s angl. [Optimal Systems Management: Translated from English.]. Moscow: Mir, 1990, 584 p.

8. Brammer K., Ziffling G. Filtr Kalmana-Byusi. Determinirovannoe nablyudenie i stohasticheskaya filtraciya [Deterministic Observation and Stochastic Filtering]. Moscow: Nauka, 1982, 199 p.

9. Jazwinski A.H. Stochastic Processes and Filtering Theory. - New York: academic press, 1970, p. 376.

10. Marpl-ml. S.L. Cifrovoj spektralnyj analiz i ego prilozheniya: Per. s angl [Digital Spectrum Analysis and its Applications]. Moscow: Mir, 1990, 584 p.

11. Shevyakova A.A. Integralnye sistemy avtomaticheskogo upravleniya silovymi ustanovkami samoletov [Integrated systems for automatic control of aircraft power plants]. Moscow: Mashinostroeniye, 1983, $283 \mathrm{p}$.

12. Kuzovkov H.T., Salychev O.S. Inercialnaya navigaciya i optimalnaya filtraciya [Inertial navigation and optimal filtering]. Moscow: Mashinostroeniye, 1982, $216 \mathrm{p}$.

13. Izerman R. Cifrovye sistemy upravleniya [Digital control systems]. Moscow: Mir, 1984, 541 p.

14. Lamanova N.G. Adaptivnoe ocenivanie vektora vyhoda SAU GTD [Adaptive estimation of the output vector of the ACS GTE]. PNRPU Aerospace Engineering Bulletin, 2000, no. 4, pp. 37-42.

15. Lamanova N.G. Pleshivyh A.S. Gribkov I.N. Fatykov A.I. Identifikaciya matematicheskoj modeli sistemy avtomaticheskogo uprav-leniya gazoturbinnogo dvigatelya [Identification of the mathematical model of the automatic control system of a gas turbine engine]. PNRPU Bulletin. Electrotechnics, Informational Technologies, Control Systems, 2019, no. 31, pp. 121-135.

16. Lamanova N.G. Analiz matematicheskoj modeli SAU GTD [Analysis of the mathematical model of ACS GTE]. PNRPU Aerospace Engineering Bulletin, 1999, no. 2, pp. 76-81.

17. Shevyakova A.A. Teoriya avtomaticheskogo upravleniya silovymi ustanovkami letatel'nyh apparatov [Theory of automatic control of power plants of aircraft]. Moscow: Mashinostroeniye, 1976, $344 \mathrm{p}$.

18. Skibin V.A., Solonin V.I., Palkin V.A. Raboty vedushchih aviastroitelnyh kompanij v obespechenie sozdaniya perspektivnyh aviacionnyh dvigatelej (analiti-cheskij obzor) [Modern methodology for creating competitive aircraft engines and the place of science in this process]. Moscow: CIAM, 2010, $673 \mathrm{p}$. 
19. Pleshivyh A.S., Zaborskih A.A., Fatykov A.I. Stend dlya ispytanij elektronnoj chasti sistem avtomaticheskogo upravleniya gazoturbinnogo dvigatelya [Test bench for the electronic part of the automatic control systems of a gas turbine engine]. PNRPU Bulletin. Electrotechnics, Informational Technologies, Control Systems, 2017, no. 22, pp. 90-102.

\section{Об авторах}

Иноземцев Александр Александрович (Пермь, Россия) - доктор технических наук, профессор, член-корреспондент Академии технологических наук Российской Федерации, член-корреспондент Российской академии наук, завкафедрой «Авиационные двигатели» ФГБОУ ВО ПНИПУ (614990, г. Пермь, ул. Профессора Поздеева, д. 13, корп. Г, к. 220); управляющий директор - генеральный конструктор АО «ОДК-Авиадвигатель» (614990, г. Пермь, Комсомольский пр., д. 93, e-mail: office@avid.ru).

Ламанова Надежда Геннадьевна (Пермь, Россия) - доцент кафедры «Прикладная математика» ФГБОУ ВО ПНИПУ (614990, г. Пермь, Комсомольский пр., д. 29, e-mail: nglaman@mail.ru).

Саженков Алексей Николаевич (Пермь, Россия) - кандидат технических наук, помощник генерального конструктора, начальник административного отдела АО «ОДК-Авиадвигатель» (614990, г. Пермь, Комсомольский пр., д. 93, e-mail: sazhenkov@avid.ru).

Грибков Игорь Николаевич (Пермь, Россия) - заместитель начальника отдела расчетно-экспериментальных работ и проектирования систем автоматического управления АО «ОДК-Авиадвигатель» (614990, г. Пермь, Комсомольский пр., д. 93, e-mail: gribkov@avid.ru).

Плешивых Артур Сергеевич (Пермь, Россия) - аспирант кафедры «Прикладная математика» ФГБОУ ВО ПНИПУ (614990, г. Пермь, Комсомольский пр., д. 29); инженер-конструктор отдела расчетно-экспериментальных работ и проектирования систем автоматического управления АО «ОДК-Авиадвигатель» (614990, г. Пермь, Комсомольский пр., д. 93, e-mail: arthur.p.s.1995@mail.ru).

\section{About the authors}

Aleksandr A. Inozemtsev (Perm, Russian Federation) - Corresponding Member of the Russian Academy of Technological Sciences, Corresponding Member of the Russian Academy of Sciences; Head of Aviation Engines Department, Perm National Research Polytechnic University (office 220, building G, 13, Professora Pozdeeva st., Perm, 614990, Russian Federation); Managing Director - General Designer, JSC "UEC-Aviadvigatel” (93, Komsomolsky av., Perm, 614990, Russian Federation, e-mail: office@avid.ru).

Nadezhda G. Lamanova (Perm, Russian Federation) - Assistant Professor of Applied Mathematics Department, Perm National Research Polytechnic University (29, Komsomolsky av., Perm, 614990, Russian Federation, e-mail: nglaman@mail.ru).

Alexey N. Sazhenkov (Perm, Russian Federation) - CSc in Technical Sciences, Assistant General Designer, Head of Administrative Department, JSC “UEC-Aviadvigatel” (93, Komsomolsky av., Perm, 614990, Russian Federation, e-mail: sazhenkov@avid.ru).

Igor N. Gribkov (Perm, Russian Federation) - Deputy Head of Design and Experimental Works and Control Systems Development Department, JSC “UEC-Aviadvigatel” (93, Komsomolsky av., Perm, 614990, Russian Federation, e-mail: gribkov@avid.ru).

Artur S. Pleshivykh (Perm, Russian Federation) - Postgraduate Student of Applied Mathematics Department, Perm National Research Polytechnic University (29, Komsomolsky av., Perm, 614990, Russian Federation); Design of Design and Experimental Works and Control Systems Development Department, JSC "UECAviadvigatel” (93, Komsomolsky av., Perm, 614990, Russian Federation, e-mail: arthur.p.s.1995@mail.ru).

Получено 11.11.2020 